\title{
Analytical chemistry for drug discovery and development
}

\begin{abstract}
Aldo Roda
Published online: 21 July 2010

(C) Springer-Verlag 2010
\end{abstract}

In recent years "pharmaceutical analysis" has benefited strongly from different technological improvements in separation sciences, modern soft impact mass spectrometry methods coupled with liquid chromatography, and the use of bioanalytical tools for molecular recognition such as antibodies and nucleic acid probes. Molecular-biology-based analytical methods for drug analysis facilitated automated high-throughput screening of new drug candidate molecules.

The overall drug development process requires robust, accurate analytical methods able to support all stages of the process: from preclinical studies to drug formulation, purity assessment, and clinical studies.

The strong quality control rules stimulated the development of new concepts in bioanalysis and instruments able to fulfill these requirements.

Pharmaceutical analysis involves a multidisciplinary analytical approach from cell-based assay to sophisticated spectroscopic technologies; all of them have to satisfy the need in drug discovery from simple organic molecules to functional proteins used as candidate drugs.

In the present issue a selection of modern analytical approaches for drug discovery and analysis as well as quality control is provided. This special issue is divided into three parts: drug analysis, quality assurance, and drug discovery.

A. Roda $(\bowtie)$

Department of Pharmaceutical Sciences,

Laboratory of Analytical and Bioanalytical Chemistry,

Alma Mater Studiorum, University of Bologna,

Via Belmeloro 6,

40126 Bologna, Italy

e-mail: aldo.roda@unibo.it
The first section of this issue is focused on the applications in pharmaceutical analysis of the main analytical techniques. Major advantages and critical issues of some promising techniques, including high-performance liquid chromatography, mass spectrometry, capillary electrophoresis, vibrational spectroscopies (infrared and Raman), X-ray diffractometry, and hyperspectral imaging techniques, are highlighted.

Besides, also the estimation of the ability of a drug to bind to plasma proteins is a crucial issue in the early drug discovery stages as well as in clinical practice. The methods of choice to obtain a complete view of these binding mechanisms as well as new approaches are discussed.

Attention is also devoted to the problem of pharmaceutical counterfeiting; it has been estimated that $10 \%$ of medicines worldwide are likely to be counterfeit and the detection of counterfeit drugs represents a challenge for public health safety. In addition, controlling and assuring the quality of Internet pharmaceuticals has become an important and challenging task for the authorities. This aspect is considered together with available analytical tools to determine the physicochemical properties of the pharmaceutical products purchased through the Internet. In addition, the feasibility of proteomics for quality-control processes in transfusion medicine is also discussed.

A brief overview of the evolution of the current pharmaceutical good manufacturing practices and process analytical technology concepts and a review of their applications in the pharmaceutical industry are provided.

The last section of the issue discusses the main analytical challenges of the drug discovery process, with a special focus on the use of circular dichroism and bioanalytical 
tools. In particular the application of fluorescence and bioluminescence resonance energy transfer for G-proteincoupled receptors is critically reviewed together with calcium-imaging-based methods.

A major part of this special issue is devoted to cellbased approaches, which are playing an ever-increasing role in drug screening routines. In fact, cell-based assays, thanks to their peculiar advantages of predictivity, possibility of automation, multiplexing, and miniaturization, seem to be appealing tools for the high demands of the early stages of the drug discovery process.

Furthermore, single-cell analysis is becoming a fundamental tool to understand cell-to-cell variability. Innovative microfluidic technologies for single-cell analysis for nextgeneration pharmacological screening, predictive toxicology, and clinical oncology are also discussed and an analytical perspective about the impact of microfluidics on the detection and characterization of biomacromolecules involved in pathological processes is provided.

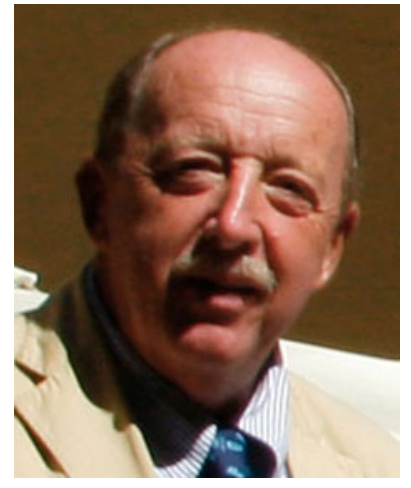

Aldo Roda

is Full Professor of Analytical Chemistry at the Alma Mater Studiorum, University of Bologna. His research activities encompass topics related to bioanalytical chemistry and biochemiluminescence, biosensors, drug analysis, and bioanalytical mass spectrometry for proteomics. He has authored more than 400 papers and book chapters published in international journals in the fields of ana-

lytical chemistry, biochemistry, physiology, medicinal chemistry, and clinical chemistry and more than 25 international patents on new bile acids and antioxidant drugs, new analytical devices and biosensors, and new luciferases. He is Editor of Analytical and Bioanalytical Chemistry and Luminescence: The Journal of Biological and Chemical Luminescence. He is Councilor on the Advisory Board of the International Society for Bioluminescence and Chemiluminescence (ISBC), a member of Accademia delle Scienze dell'Istituto di Bologna, and a member of the councilor board of the Istituto Nazionale di Biostrutture e Biosistemi (INBB) for biotechnology. 